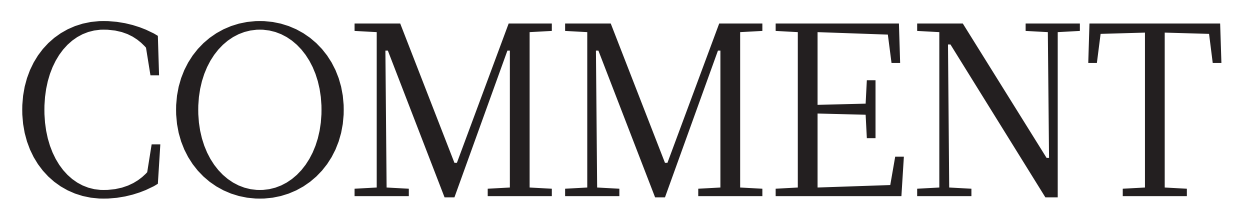

\begin{tabular}{|c|c|c|c|}
\hline $\begin{array}{l}\text { SUSTAINABILITY The stories } \\
\text { that inspire tomorrow's } \\
\text { environmentalists p.434 }\end{array}$ & $\begin{array}{l}\text { Z0oLOGY From pheromones to } \\
\text { faeces: the world through } \\
\text { the nose of a dog } \mathbf{p . 4 3 5}\end{array}$ & $\begin{array}{l}\text { PoLICY Recovery of Canadian } \\
\text { cod is too small and slow } \\
\text { to ramp up fishing } \mathbf{p . 4 3 6}\end{array}$ & $\begin{array}{l}\text { CLIMATE CHANGE Geoengineering } \\
\text { glaciers could do more harm } \\
\text { than good } \mathbf{p . 4 3 6}\end{array}$ \\
\hline
\end{tabular}

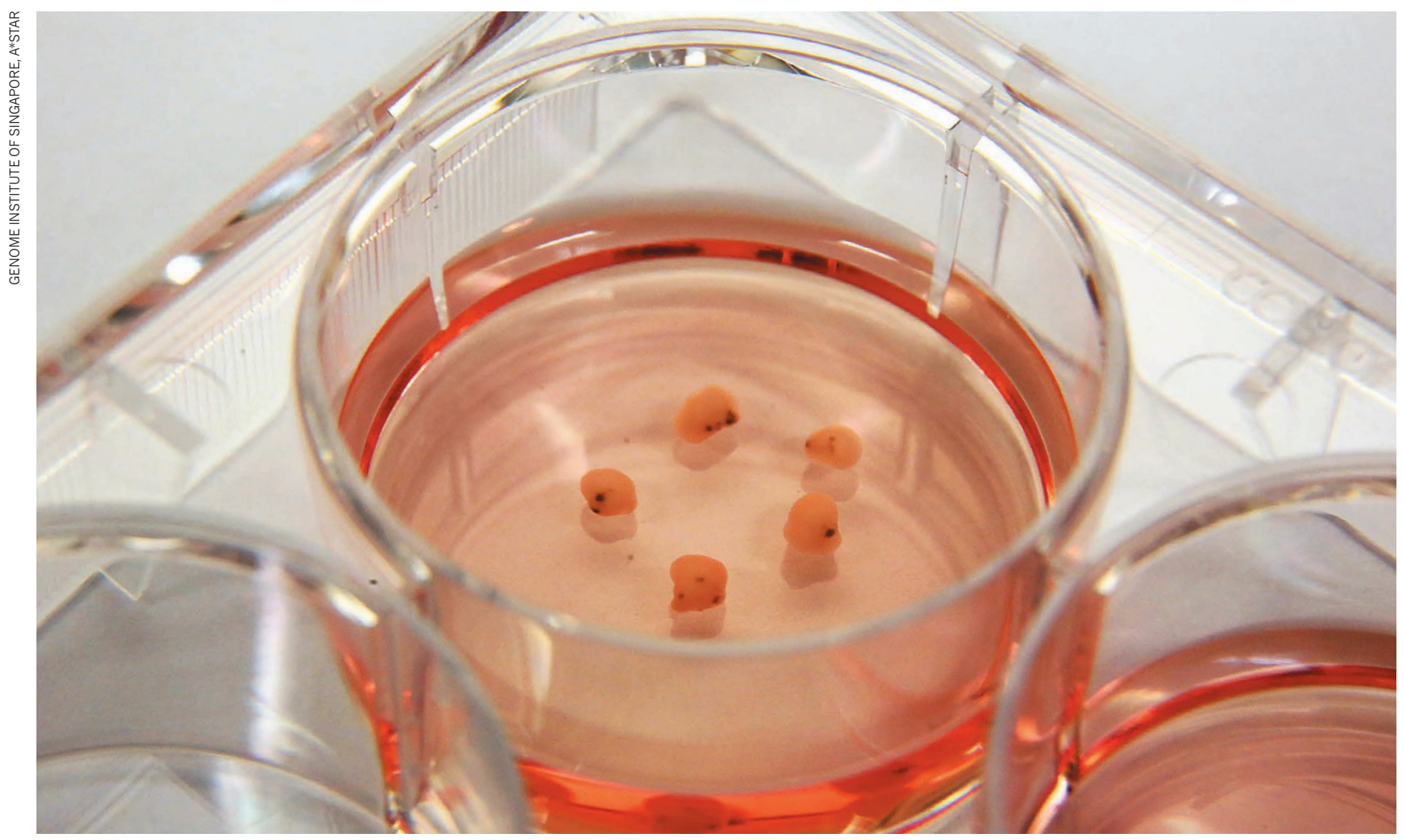

Simplified 3D brain organoids can be grown in a dish using human stem cells as the starting material.

\title{
The ethics of experimenting with human brain tissue
}

Difficult questions will be raised as models of the human brain get closer to replicating its functions, explain Nita A. Farahany, Henry T. Greely and 15 colleagues.

$\mathrm{I}$ f researchers could create brain tissue in the laboratory that might appear to have conscious experiences or subjective phenomenal states, would that tissue deserve any of the protections routinely given to human or animal research subjects?

This question might seem outlandish. Certainly, today's experimental models are far from having such capabilities. But various models are now being developed to better understand the human brain, including miniaturized, simplified versions of brain tissue grown in a dish from stem cells - brain organoids ${ }^{1,2}$. And advances keep being made.

These models could provide a much more accurate representation of normal and abnormal human brain function and development than animal models can (although animal models will remain useful for many goals). In fact, the promise of brain surrogates is such that abandoning them seems itself unethical, given the vast amount of human suffering caused by neurological and psychiatric disorders, and given that most therapies for these diseases developed in animal models fail to work in people. Yet the closer the proxy gets to a functioning human brain, the more ethically problematic it becomes.

There is now a need for clear guidelines for research, albeit ones that can be $>$ 
adapted to new discoveries. This is the conclusion of many neuroscientists, stemcell biologists, ethicists and philosophers - ourselves included - who gathered in the past year to explore the ethical dilemmas raised by brain organoids and related neuroscience tools. A workshop was held in May 2017 at the Duke Initiative for Science \& Society at Duke University in Durham, North Carolina, with limited support from the US National Institutes of Health (NIH) BRAIN Initiative. A similar US meeting was held last month on related topics.

Here we lay out some of the issues that we think researchers, funders, review boards and the public should discuss as a first step to guiding research on brain surrogates.

\section{SAFE SURROGATES}

Three classes of brain surrogate offer researchers a way to investigate how the living human brain works, without the need for potentially risky - if not ethically impossible - procedures in people.

Organoids. Brain organoids can be produced much as other $3 \mathrm{D}$ multicellular structures resembling eye, gut, liver, kidney and other human tissues have been built ${ }^{2-4}$. By adding appropriate signalling factors, aggregates of pluripotent stem cells (which have the ability to develop into any cell type) can differentiate and self-organize into structures that resemble certain regions of the human brain ${ }^{5-7}$.

Investigators use different approaches. They might coax pluripotent stem cells to turn into specific populations of neural cells, such as those specific to a particular brain region. Or they can allow the pluripotent cells to differentiate on their own, in which case both neural cells and other cell types might be generated ${ }^{2}$. Brain organoids resembling particular brain regions can even be combined into 'brain assembloids' to enable researchers to study the formation of neural circuits and cellular interactions between different regions ${ }^{8}$.

Compared with 2D sheets of neural cells in a dish, the 3D structures last longer (for around two years ${ }^{9}$ ) and can consist of more types of cell. They also mimic key features of developing brains. For instance, in later stages of fetal development, the cerebral cortex switches from generating neurons to creating glial cells (the various other cell types in the brain that nourish, surround and protect neurons). This process can be captured in brain organoids, allowing investigators to gain insights that would be experimentally and ethically extremely challenging, if not ethically unacceptable, to obtain from developing brains.

Already, researchers have deployed brain organoids to investigate neurodevelopmental alterations in people with autism spectrum disorders ${ }^{8,10}$ or schizophrenia ${ }^{11}$, and to study the unusually small brain

size (microcephaly) seen in some babies infected with the Zika virus before birth ${ }^{12}$.

Brain organoids have limitations. They lack certain cell types, such as microglia and cells that form blood vessels. Today, the largest organoids are about 4 millimetres in diameter and contain only about 2 million to 3 million cells. An adult human brain measures roughly 1,350 cubic centimetres, and is made up of 86 billion neurons and a similar number of non-neuronal cells. Moreover, so far, brain organoids have received sensory input only in primitive form, and connections from other brain regions are limited.

Given such constraints, the possibility of organoids becoming conscious to some degree, or of acquiring other higherorder properties, such as the ability to feel distress, seems highly remote. But organoids are becoming increasingly complex. Indeed, one of us (P.A.) recorded neural activity from an organoid after shining light on a region where cells of the retina had formed together with cells of the brain. This illustrated that an external stimulus can result in an organoid response $\mathrm{e}^{13}$.

Ex vivo brain tissue. Another type of model involves slices of brain tissue that have been removed from individuals during some surgical procedure, for example to treat seizures.

For more than a century, researchers have studied brain cells in tissue extracted from patients undergoing surgery, or from people who have died. But technological advances, including in imaging and in the techniques used to preserve

\section{"The possibility of organoids becoming conscious to some degree seems highly remote."}

the functional properties of brain tissues in the lab (exvivo), could make this approach considerably more powerful.

When tissue from the neocortex or hippocampus regions is removed to treat a pathology, such as epilepsy or cancer, the piece removed is typically the size of a sugar cube (about 1-4 cubic centimetres), although it can sometimes be much bigger. That piece is then generally cut into slices, the functional properties of which can be preserved for weeks.

Using these slices, researchers can measure the synaptic and other properties of neurons in intact brain circuits; map the 3D morphology of circuits; and extract and analyse cellular RNA to probe gene expression. They can also manipulate the firing of specific neurons using optogenetics, which could enable them to analyse in more detail the functional properties of human brain circuits. (Optogenetics uses light to track or selectively activate neurons that have been genetically modified to express a light-sensitive protein.)

Currently, ex vivo brain tissue does not have sensory inputs. And with outbound connections severed, isolated tissues can't communicate with other regions of the brain, or generate motor outputs. Thus, the possibility of consciousness or other higher-order perceptive properties emerging seems extremely remote.

Chimaeras. The third class of experimental brain model involves the transplantation of human cells, derived in vitro from pluripotent stem cells, into the brains of animals such as rodents. This can be done while the animal fetus is developing or after the animal is born. Such chimaeras are generated to provide a more physiologically natural environment in which the human cells can mature.

Neuroscientists have transplanted human glial cells into mice, for instance, and found that the animals perform better in certain tasks involving learning. Researchers have also injected human stem cells into early-stage pig embryos, and then transferred the embryos into surrogate sows, where they've been allowed to develop until the first trimester. More than 150 of the embryos developed into chimaeras; in these embryos, about 1 in 10,000 cells in the precursors of hearts and livers were human.

In principle, chimaeras could help researchers to better understand human illnesses and the effects of drug treatments. Labs have developed human-mouse chimaeras to shed light on Parkinson's disease, for example.

Some groups have even successfully transplanted human brain organoids into rodents, where they have become supported by blood vessels (vascularized) ${ }^{14}$. The provision of a blood supply is an essential step in enabling organoids to grow larger than their current achievable size. But the size of rodent models restricts the degree to which human brain organoids can grow within them.

\section{ISSUES TO CONSIDER}

Currently, if research on human tissue occurs outside a living person, only the processes of obtaining, storing, sharing and identifying the tissue fall under the regulations and guidelines that limit what interventions can be conducted on people. As brain surrogates become larger and more sophisticated, the possibility of them having capabilities akin to human sentience might become less remote. Such capacities could include being able to feel (to some degree) pleasure, pain or distress; being able to store and retrieve memories; or perhaps even having some perception of agency or awareness of self.

Could studies involving brain tissue that has been removed from a living 


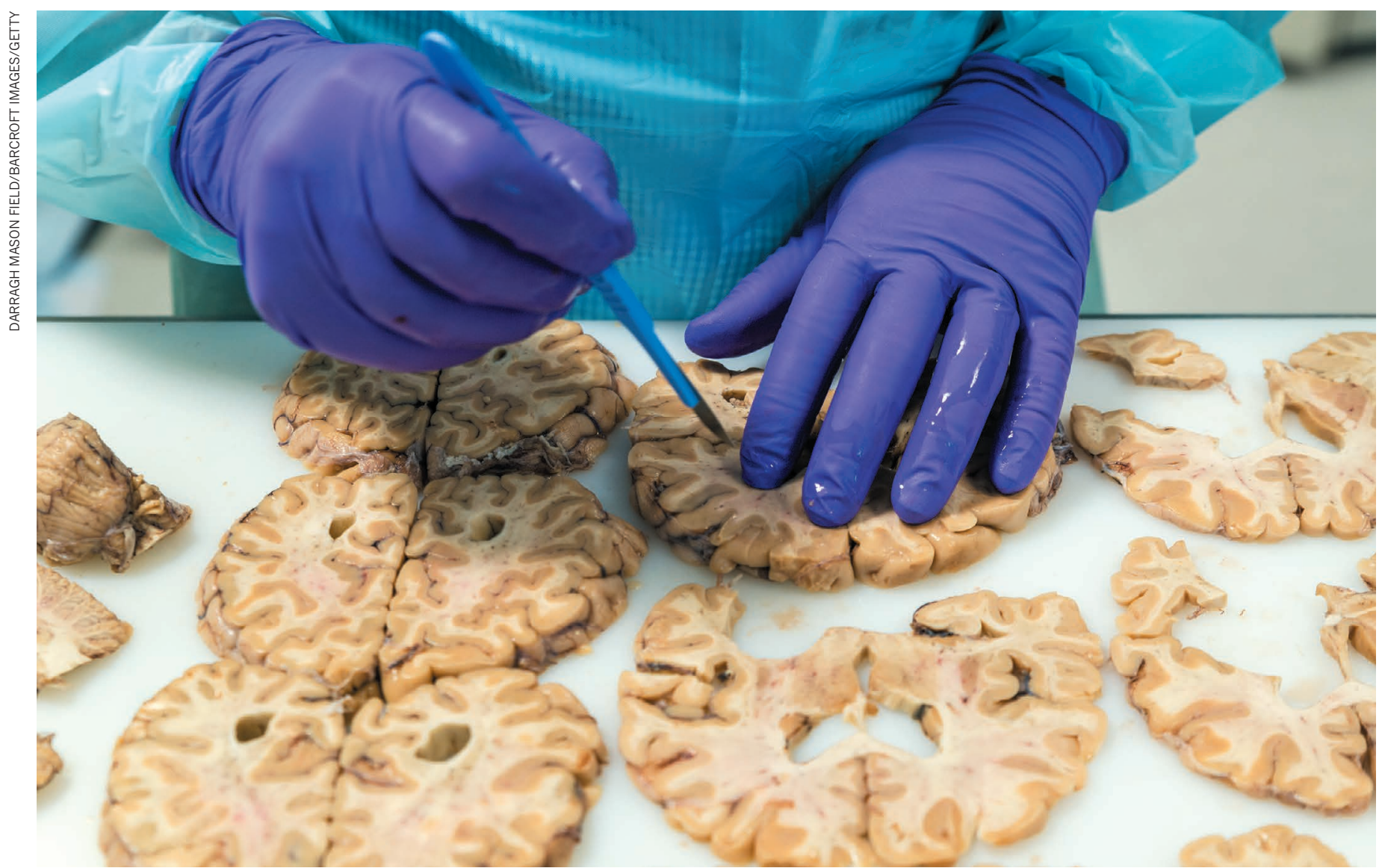

A researcher dissects slices of human brain tissue.

person or corpse provide information about the person's memories, say? Could organisms that aren't 'biologically human' ever warrant some degree of quasi-human or human moral status?

In the light of such possibilities, here we lay out some of the issues that we think civil society, researchers, ethicists, funders and reviewers ought now to be considering.

Metrics. Is it even possible to assess the sentient capabilities of a brain surrogate? What should researchers measure? If appropriate metrics can be developed, how do investigators decide which capabilities are morally concerning?

Neuroscientists have made considerable progress when it comes to identifying the neural correlates of consciousness ${ }^{15}$. Yet the signals for consciousness or unconsciousness detected in a living adult - using electroencephalography (EEG) electrodes, for example - don't necessarily translate to infants, animals or experimental brain surrogates. Without knowing more about what consciousness is and what building blocks it requires, it might be hard to know what signals to look for in an experimental brain model ${ }^{15}$.

With regard to human-animal chimaeras, researchers are already dealing with beings that have some form of consciousness. Here, the need to establish what measures to base protections on (both for the animal and the human subject) is more pressing. One possibility is for researchers to use anaesthetics or other methods to maintain comatose-like brain states. Perhaps certain brain functions or a prespecified level of brain activity, signalling a lack of capacity, could be used to delineate ethically justifiable research.

Human-animal blurring. Researchers have already produced mice with rat pancreases by injecting rat pluripotent stem cells into mouse embryos. The same approach could one day enable the production of human organs in other animals ${ }^{16}$.

How do we define the boundaries of this research? What implications might such boundaries have for vascularizing brain organoids, or for growing neural tissue in animals? Is the production of a human heart in a pig's body acceptable, for instance, but not the production of a brain from human cells?

We believe that decisions about which kinds of chimaera are permitted, or about whether certain human organs grown in animals make animals 'too human-like', should ultimately be made on a case-bycase basis - taking into account the risks, benefits and people's diverse sensitivities.

Death. Do ex vivo human brain models challenge our understanding of life and death? What implications might such models have for the legal definition of death, and what are the implications for decisions tied to this definition, such as organ donation?

The advent of tracheal positive-pressure ventilation in the 1950s and cardiopulmonary resuscitation (CPR) in the 1960s led to the concept of brain death. Beginning in the 1960 s, a person whose brain had completely and irreversibly ceased to function could be declared dead, even if they still had a heartbeat.

Any emerging technologies that could restore lost functionality to a person's brain could potentially undermine the diagnosis of brain death, because the cessation of brain function might no longer be permanent and irreversible. But a distinction here is important: technologies that would restore a few neurons or certain limited kinds of brain activity would not restore clinical functionality of the brain and so would not raise this concern.

Consent. Is the standard process of obtaining informed consent adequate for research using human brain cells or tissue, or developing brain surrogates from induced pluripotent stem cells?

Currently, researchers using pluripotent stem cells or brain tissues generally disclose their plans to donors in broad terms. Given how much people associate their experiences and sense of self with their brains, more transparency and assurances 
could be warranted. Donors might wish to deny the use of their stem cells for the creation of, say, human-animal chimaeras.

This targeted approach is used in other contexts. When people undergoing in vitro fertilization procedures choose to donate excess embryos to research, for instance, they are assured that these will not be used to create a baby.

Stewardship. Is there a point at which we should be concerned about the welfare of brain surrogates or chimaeras, such that assigning someone loosely akin to a guardian or decision-maker for the brain surrogate might be warranted, beyond the researchers involved? Such an arrangement would be similar to the appointment of a guardian ad litem in custody disputes involving children in the United States (someone besides the parents who can represent the child's interests).

Ownership. Who, if anyone, should 'own' ex vivo brain tissue, brain organoids or chimaeras?

At present, brain tissue samples are owned by the researchers or organizations collecting the tissue or doing the science. If significant developments in the field one day lead us to regard any of these brain surrogates as having greater moral status than we would currently give them, might greater privileges and protections be appropriate?

Post-research handling. How should human brain tissue be disposed of, or handled at the end of an experiment?

Today, brain organoids or ex vivo brain tissue are destroyed following standard practices for disposing of all tissues. But if researchers develop mice, say, with some advanced cognitive capacities, should those animals be destroyed or given special treatment at the end of a study? Already certain animals, such as chimpanzees, enter sanctuaries to live out the remainder of their lives after researchers have finished working with them in laboratories.

Data. Should there be special requirements for data sharing, collaboration and legacy use of brain tissue?

The unique benefits and risks of sharing data obtained from such tissues will need to be considered. Ex vivo human brain tissue could reveal sensitive information for instance, about a person's memories or disease status. Equally, there could be more value in sharing such information, because of the difficulty of obtaining human brain tissue. In some cases, certain features of the data might need to be stripped out, or the extent of sharing limited.

Geneticists have long grappled with similar issues for people's genomic information; some of their approaches could be applied to brain research.

\section{ETHICS EFFORTS}

Various efforts are already tackling the ethics of advances in neuroscience ${ }^{17}$. When the BRAIN Initiative was announced in 2013, the Presidential Commission for the Study of Bioethical Issues was charged with evaluating ethics, and produced a two-volume report in response ${ }^{18,19}$. The European Commission's Human Brain Project has a major ethics component, and the NIH BRAIN Initiative has a neuroethics division.
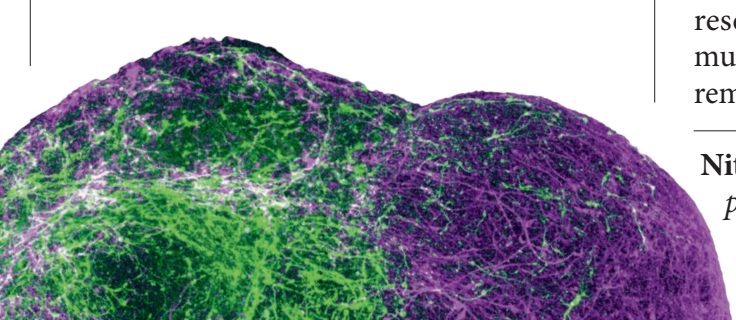

A. Farahany is professor of law and

philosophy at Duke University, director of the Duke Initiative for Science \& Society, Duke University, Durham, North Carolina, USA. Henry T. Greely is professor of law, director of the Center for Law and the Biosciences, and director of the Stanford Program in Neuroscience and Society at Stanford University, California, USA.

Steven Hyman, Christof Koch, Christine Grady, Sergiu P. Pașca, Nenad Sestan, Paola Arlotta, James L. Bernat, Jonathan Ting, Jeantine E. Lunshof, Eswar P. R. Iyer, Insoo Hyun, Beatrice H. Capestany, George M. Church, Hao Huang, Hongjun Song. e-mail:farahany@duke.edu

1. Quadrato, G., Brown, J. \& Arlotta, P. Nature Med. 22, 1220-1228 (2016).

2. Pasca, S. P. Nature 553, 437-445 (2018).

3. Arlotta, P. Nature Meth. 15, 27-29 (2018).

4. Eisenstein, M. Nature Meth. 15, 19-22 (2018).

5. Lancaster, M. A. \& Knoblich, J. A. Nature Protoc. 9, 2329-2340 (2014).

6. Qian, X. et al. Cell 165, 1238-1254 (2016).

7. Kadoshima, T. et al. Proc. Natl Acad. Sci. USA 110, 20284-20289 (2013)

8. Birey, F. et al. Nature 545, 54-59 (2017).

9. Sloan, S. A. et al. Neuron 95, 779-790 (2017).

10.Mariani, J. et al. Cell 162, 375-390 (2015).

11.Ye, F. et al. Neuron 96, 1041-1054 (2017).

12.Qian, X., Nguyen, H. N., Jacob, F., Song, H. \& Ming, G. L. Development 144, 952-957 (2017)

13.Quadrato, G. et al. Nature 545, 48-53 (2017).

14. Mansour, A. A. et al. Nature Biotechnol. https:// doi.org/10.1038/nbt.4127 (2018).

15. Koch, C., Massimini, M., Boly, M. \& Tononi, G. Nature Rev. Neurosci. 17, 307-321 (2016).

16.Bourret, R. et al. Stem Cell. Res. Ther. 7, 87 (2016).

17. Greely, H. T., Ramos, K. M. \& Grady, C. Neuron 92 637-641 (2016).

18.Gray Matters: Integrative Approaches for Neuroscience, Ethics, and Society (Presidential Commission for the Study of Bioethical Issues, 2014); available at https://go.nature. $\mathrm{com} / 2 \mathrm{qvm} 83 \mathrm{f}$

19. Gray Matters: Topics at the Intersection of Neuroscience, Ethics, and Society (Presidential Commission for the Study of Bioethical Issues, 2015); available at https://go.nature. $\mathrm{com} / 2 \mathrm{vdq} \times 5 \mathrm{j}$

A full list of author affiliations accompanies this Comment online (see go.nature.com/2hz3ogu). 